\title{
\%
}

Joaquín María Núñez Varo*

María del Mar Gómez González**

\section{EL VALOR ESTRATÉGICO DE LA EVALUACIÓN EN ICEX}

La estrategia de evaluación iniciada en 2011 por ICEX tenía como objetivo prioritario la mejora de la calidad del servicio que se ofrece a la empresa internacionalizada, basándonos para ello en análisis metódicos y conclusiones rigurosas. Esta línea de trabajo contemplaba desde el principio la opción de enriquecer el enfoque clásico de evaluación con técnicas de ciencia de datos. Y gracias a esa evolución, toda la evidencia y el conocimiento adquiridos durante estos años nos abren de forma natural la oportunidad de poner en marcha un sistema de recomendación que nos permita conocer y poder proponer, con suficiente precisión y de manera anticipada, las actividades e instrumentos de apoyo que puedan resultar más apropiadas para nuestro cliente, de forma que se optimice el valor y la gestión de la acción pública. Y este gran paso promete ser tan solo el comienzo de un largo y fructífero camino que se inicia gracias a la unión estratégica entre el conocimiento fruto de la evaluación y la creciente transformación digital por la que estamos apostando.

Palabras clave: evaluación de impacto, evaluación de políticas públicas, inteligencia artificial, internacionalización empresarial, métodos cuantitativos.

Clasificación JEL: C02, C44, C52, C93, L53.

\section{Introducción}

La Unidad de Evaluación de ICEX se creó en 2011 con la finalidad de conocer qué utilidad tenían los diferentes apoyos que prestaba la institución. Sin embargo, y desde el principio,

\footnotetext{
* Analista del Departamento de Evaluación de ICEX España Exportación e Inversiones. Profesor Asociado de la Universidad Carlos III de Madrid. Miembro del Comité de Asesoramiento Científico del Centro de Investigación en Economía Digital de la Universidad Autónoma de Madrid.

** Directora Adjunta de Sostenibilidad y Evaluación de ICEX España Exportación e Inversiones. Profesora Colaboradora en el Máster de Comercio Exterior de la Universidad Carlos III de Madrid.

Versión de abril de 2020.

DOI: https://doi.org/10.32796/bice.2020.3123.7003
}

no quisimos limitarnos a la realización de meros trabajos teóricos, sino que nuestro objetivo era que las conclusiones extraídas proporcionaran evidencia robusta para contribuir a la mejora continua de nuestro apoyo a la internacionalización de la empresa.

Para conseguir este objetivo tuvimos claro que había que enriquecer las metodologías clásicas de evaluación con analítica, término con que se agrupan técnicas de ciencia de datos que se vienen aplicando desde hace al menos una década en marketing digital.

El presente artículo tiene por objetivo dar a conocer la estrategia seguida por ICEX en $D$ 
materia de evaluación, sin cargar demasiado el texto con la parte técnica, que se encuentra explicada en Núñez (2017), donde remitimos al lector interesado. Si bien reproduciremos alguna parte de ese trabajo, nuestro interés es presentar las fases de nuestra estrategia de evaluación y las preguntas que nos hemos ido planteando, ofrecer los resultados obtenidos en cada caso y las posibilidades que esto nos abre de cara al futuro, pues estamos poniendo en marcha un sistema de recomendación capaz de proponer con suficiente precisión y de manera anticipada las actividades e instrumentos de apoyo que puedan solventar las necesidades de nuestro cliente (la empresa durante su proceso de internacionalización) en términos de maximizar tanto la efectividad del apoyo como el valor de la acción pública.

Un proyecto de esta índole no se podría haber planteado sin la conjunción de tres elementos que se han venido impulsando en ICEX: la apuesta por un profundo y detallado conocimiento extraído en los ejercicios de evaluación, el proceso de transformación y digitalización de la gestión del cliente que ha llevado a cabo la Dirección de Desarrollo Digital y la modernización y securización de los sistemas de Tecnologías de Información.

\section{Fases en la estrategia de evaluación}

Cuando empezamos en 2011, constatamos que no disponíamos de las fuentes de datos necesarias para poder llevar a cabo ninguna evaluación con datos administrativos, y también carecíamos de información de empresas españolas que no hubieran sido usuarias de apoyos de ICEX y que pudieran ser candidatos razonables para conformar un grupo de control válido con el que calcular el contrafactual, es decir, lo que habría ocurrido en caso de no haber existido la intervención.

Por este motivo, nos planteamos las siguientes fases en nuestro proyecto de evaluación.

Una primera fase consistente en poder establecer los modelos operativos de los diferentes programas de apoyo a la empresa a partir de una completa reconstrucción de las lógicas de las distintas intervenciones, lo que en sí constituye una evaluación teórica del diseño de cada tipo de apoyo. La reconstrucción de estas lógicas sacó a la luz que tan solo podríamos considerar que un apoyo había tenido éxito si estaban alineados los siguientes aspectos: necesidades de la empresa, producto o servicio entregado por ICEX y resultados que dicho producto o servicio permite alcanzar a la empresa en caso de que esta haga uso del mismo. Diseñamos a continuación un cuestionario para poder capturar información de estos aspectos, que incluía no solo las características más relevantes de las empresas y los efectos percibidos en doce variables de mejoras de competitividad, que llamamos internamente variables de resultado (resultado a corto plazo) e impacto (resultado a medio plazo), sino también sus preferencias de apoyo en el tipo de servicio en cuestión. Al carecer de grupos de control reales, utilizamos como técnica de estimación del impacto la técnica de la pregunta implícita, que utiliza al propio grupo de tratamiento como su grupo de control. Explicaremos el desarrollo de esta primera fase en profundidad en este artículo, y referimos al lector interesado por los detalles técnicos a Núñez (2017).

Una segunda fase consistente en identificar grupos de control para cada tipología de empresa y variables reales que aterricen los $D$ 
aspectos de competitividad global donde nuestra primera fase de evaluación ha mostrado efecto en la empresa o atribución de esta al apoyo público. Esta etapa se encuentra en desarrollo en la actualidad de forma interna ${ }^{1}$.

Como tercera fase de nuestra estrategia, planteamos que el mejor indicador del impacto a largo plazo de las políticas públicas de apoyo a la internacionalización de la empresa es el retorno de la inversión pública, vía impuestos, asociado a aquellos resultados en las empresas que pueden ser atribuidos sin error a nuestro apoyo mediante técnicas de evaluación. Entendemos que esta tercera fase se debería llevar a cabo teniendo en cuenta el tratamiento no solo de los servicios de un único organismo, sino el apoyo agregado de todas las instituciones públicas en el ámbito de la internacionalización, entre las que ICEX puede ser la más relevante, aunque con claridad no la única, con el objetivo de disponer de una visión integral y obtener conclusiones transversales.

De forma independiente al desarrollo de esta tercera fase, que ICEX no puede realizar en solitario, el conocimiento obtenido hasta ahora en la primera fase y el que vayamos obteniendo en la segunda nos permiten, desde ya, poder mejorar el servicio que damos a la empresa. A continuación, pasamos a exponer en detalle en qué ha consistido la primera fase de nuestra estrategia de evaluación. Debido al límite en la extensión de este artículo, nos centraremos más en las evaluaciones de impacto

1 Una primera aproximación a este trabajo se ha realizado gracias al convenio de colaboración entre el Banco de España, ICEX, INE y la Secretaría de Estado de Comercio para la evaluación del Plan de Internacionalización de la Empresa Española, donde uno de los autores (Núñez) se ha encargado de la evaluación de impacto del apoyo de ICEX. Los primeros resultados obtenidos, que todavía no se pueden publicar, confirman todas las conclusiones alcanzadas hasta ahora en nuestras evaluaciones. En particular, se ha utilizado una combinación de dos técnicas: emparejamiento exacto forzado (coarsened exact matching, CEM), donde los grupos han sido determinados mediante un clusterizado de K-medias, y diferencias en diferencias dentro de cada uno de los grupos determinados anteriormente. y nos limitaremos a ofrecer algunos apuntes sobre las evaluaciones de necesidades.

Para centrar al lector menos familiarizado, una evaluación de impacto tiene por fin dar respuesta a dos preguntas:

1. ¿Ha habido algún efecto en los beneficiarios tras la intervención?

2. ¿Se puede atribuir un vínculo causal entre la intervención y los efectos observados?

La respuesta a ambas preguntas se denomina el impacto de la intervención, que expresa el grado de cumplimiento de los objetivos en la población que se quería atender.

Con este tipo de evaluación tratamos de contrastar empíricamente si el apoyo público de ICEX ha tenido algún efecto en las empresas beneficiarias. Hemos desarrollado una metodología que permite identificar efectos a lo largo del tiempo, y nuestro sistema de atribución de resultados ha recibido el aval del International Trade Centre (ITC) dentro del conjunto de mejores prácticas internacionales en materia de evaluación.

Por su parte, una evaluación de necesidades trata de identificar las necesidades de los beneficiarios. En nuestro caso, el objetivo principal de este tipo de evaluación es investigar aquellas carencias de nuestras empresas cliente en algún ámbito de actuación de ICEX, así como el tipo más adecuado de apoyo público que precisarían para poder cubrirlas. Realizamos en primer lugar estudios cualitativos para extraer ideas que permitan afinar algún producto o servicio ya prestado o crear algún producto o servicio ex novo, y realizamos tests clásicos de producto para contrastar su adecuación y utilidad. A continuación, aplicamos una metodología cuantitativa propia $\triangle$ 
de análisis de necesidades adaptable a cada tipo de apoyo concreto que nos permite estimar el interés de la empresa en las mejoras propuestas para atender las necesidades identificadas, así como la intención de precio de los clientes a la hora de contratar los servicios o productos propuestos.

\section{La misión de ICEX}

ICEX tiene como misión apoyar a las empresas, la formación de profesionales y la atracción de inversiones extranjeras para incrementar la internacionalización de la economía española y acelerar un crecimiento económico más inclusivo y sostenible, con empleo de calidad.

La pregunta que surge de forma inmediata es cómo se mide la internacionalización. El desarrollo internacional de una empresa tiene cuatro componentes: exportación de bienes, exportación de servicios, inversión directa en el extranjero e ingresos por rentas de la inversión directa. En 2018, las cifras agregadas para España de estas magnitudes, según el Banco de España, se muestran en la Tabla 1.

En el fondo, todas esas magnitudes son el reflejo de distintos resultados derivados del concepto denominado competitividad en el entorno global, concepto igualmente difícil de medir. Inspirados por el ejemplo del UKTI británico, que, enfrentado a la misma dificultad de acceso a los datos, había diseñado un sistema de encuestas en el que preguntaban a las empresas por cuestiones relativas a su competitividad en el entorno global, comprobamos que podíamos captar el efecto de nuestras acciones sin necesidad de recurrir a registros oficiales o de tipo administrativo, al menos en un principio. Así, diseñamos en 2011 una primera encuesta para nuestra primera evaluación (Servicios Personalizados 2011) que hemos ido mejorando y enriqueciendo hasta que en 2014 tomó una forma estándar con un núcleo fijo y ciertos bloques específicos que dependían del programa a evaluar. El núcleo fijo, que se muestra en la Tabla 2, permite la comparabilidad entre programas, y los bloques específicos de cada programa permiten profundizar en aspectos concretos de cada intervención.

En esta primera fase de nuestro programa de evaluación, nuestro interés se ha centrado en hallar el modelo operativo, como mínimo a tres años vista tras la finalización del apoyo de cada uno de nuestros más de cien tipos de apoyo, sustentado en datos recabados a partir de encuestas de una muestra representativa de las empresas cliente. Cuando en dichas evaluaciones surgía como conclusión que algún tipo de apoyo no procuraba en las empresas el resultado perseguido, planteamos evaluaciones de necesidades para identificar cómo debíamos mejorar dicho $\triangleright$

TABLA 1

MAGNITUDES AGREGADAS DEL RESULTADO DE LA INTERNACIONALIZACIÓN DE LA EMPRESA ESPAÑOLA EN 2018 (Valores en millones de euros. Porcentajes con respecto al total)

\begin{tabular}{|c|c|c|c|c|}
\hline & $\begin{array}{l}\text { Exportación } \\
\text { de bienes }\end{array}$ & $\begin{array}{l}\text { Exportación } \\
\text { de servicios }\end{array}$ & $\begin{array}{l}\text { Inversión directa según } \\
\left.\text { principio direccional ( }{ }^{\star}\right)\end{array}$ & $\begin{array}{c}\text { Rentas de la } \\
\text { inversión directa }\end{array}$ \\
\hline Volumen & 290.255 & 131.913 & 25.432 & 32.485 \\
\hline Porcentaje & 60,46 & 27,48 & 5,30 & 6,77 \\
\hline
\end{tabular}




\section{EL VALOR ESTRATÉGICO DE LA EVALUACIÓN EN ICEX}

TABLA 2

BLOQUES FIJOS DE LAS ENCUESTAS DE IMPACTOY RESULTADO DE ICEX

\begin{tabular}{|c|c|c|}
\hline Bloque & Subbloque & Pregunta \\
\hline \multirow{3}{*}{$\begin{array}{l}\text { EFECTOS para la } \\
\text { productividad y } \\
\text { competitividad de la } \\
\text { empresa }\end{array}$} & $\begin{array}{l}\text { Cambios de comportamiento } \\
\text { (resultado) }\end{array}$ & $\begin{array}{l}\text { Introducción de mejoras } \\
\text { Confianza en explotación de nuevos mercados } \\
\text { Incremento de gasto en I+D }\end{array}$ \\
\hline & Nuevas habilidades (resultado) & $\begin{array}{l}\text { Adquisición de nuevas ideas } \\
\text { Mejora en la estrategia de marketing } \\
\text { Mejora de habilidades de investigación } \\
\text { Concienciación sobre intangibles }\end{array}$ \\
\hline & $\begin{array}{l}\text { Operativa comercial (impacto o } \\
\text { resultado a largo plazo) }\end{array}$ & $\begin{array}{l}\text { Entrada en nuevos mercados } \\
\text { Mantenimiento de clientes en mercado explotados } \\
\text { Efecto positivo en la facturación } \\
\text { Efecto significativo en contratación/mantenimiento personal } \\
\text { Efecto positivo en la cuenta de pérdidas y ganancias }\end{array}$ \\
\hline \multicolumn{2}{|c|}{ Valor añadido del apoyo público } & $\begin{array}{l}\text { ADICIONALIDAD reconocida de la política pública } \\
\text { BENEFICIOS vs. coste de la participación }\end{array}$ \\
\hline \multicolumn{2}{|c|}{$\begin{array}{l}\text { EXPECTATIVAS antes de entrar al programa y } \\
\text { LOGROS conseguidos al término }\end{array}$} & $\begin{array}{l}\text { Ajuste del plan de negocio, orientación estratégico-operativa del } \\
\text { proyecto original por parte del gestor ICEX } \\
\text { Mejora del conocimiento o acercamiento a las instituciones públicas } \\
\text { de apoyo al comercio internacional y su red en el exterior } \\
\text { Otros aspectos específicos para cada programa }\end{array}$ \\
\hline \multicolumn{2}{|c|}{ PREFERENCIAS de APOYOS PÚBLICOS } & $\begin{array}{l}\text { Apoyo económico } \\
\text { Acceso efectivo a información comercial relevante } \\
\text { Acceso efectivo a clientes y socios en el exterior } \\
\text { Mejora del perfil y credibilidad en el exterior } \\
\text { Acciones presenciales de promoción sectorial } \\
\text { Campañas de imagen del sector sin presencia de la empresa }\end{array}$ \\
\hline
\end{tabular}

apoyo o si se requería un tipo de apoyo nuevo para conseguir el objetivo fijado. Las Tablas 3 , 4,5 y 6 muestran las evaluaciones realizadas desde 2011 hasta la fecha de publicación de este artículo, así como aquellas en marcha o previstas.

TABLA 3

EVALUACIONES DE IMPACTO REALIZADAS ENTRE 2011 Y 2019

\begin{tabular}{|c|c|c|c|c|}
\hline Año & Campaña & Muestra & Universo & Tasa de respuesta \\
\hline 2011 & Servicios personalizados & 605 & 1.417 & $42,70 \%$ \\
\hline 2012 & Programa de Apoyo a Proyectos de Inversión Productiva (PAPI) & 176 & 399 & $44,11 \%$ \\
\hline 2012 & Planes sectoriales & 1.995 & 7.056 & $28,27 \%$ \\
\hline 2013 & Plan de Iniciación a la Promoción Exterior (PIPE) & 747 & 1.265 & $59,05 \%$ \\
\hline 2013 & Planes generales & 1.152 & 2.637 & $43,69 \%$ \\
\hline 2015 & Planes de empresa & 508 & 952 & $53,36 \%$ \\
\hline 2016 & Servicios personalizados & 1.953 & 4.929 & $39,62 \%$ \\
\hline \multicolumn{2}{|c|}{ Totales } & 7.136 & 18.655 & $38,25 \%$ \\
\hline
\end{tabular}


TABLA 7

LÓGICA DE LA INTERVENCIÓN DEL APOYO GENÉRICO DE ICEXY PREGUNTAS RELEVANTES

\begin{tabular}{|c|c|c|}
\hline Entidad & Variables & Preguntas relevantes \\
\hline Beneficiario & Características de la empresa & ¿Quiénes son? \\
\hline Tratamiento & Apoyo de ICEX & $\begin{array}{l}\text { ¿En qué consiste? } \\
\text { ¿Cómo nos utilizan las empresas? } \\
\text { ¿Siguen alguna estrategia? }\end{array}$ \\
\hline Producto & $\begin{array}{l}\text { Servicio prestado o producto entregado } \\
\text { (cuestionario de calidad y satisfacción) }\end{array}$ & $\begin{array}{l}\text { ¿Qué ofrecemos? } \\
\text { ¿Qué necesitan? }\end{array}$ \\
\hline Resultado & $\begin{array}{l}\text { Mejora de competitividad } \\
\text { (mayor valor añadido de la oferta española) } \\
\text { Exportación } \\
\text { Inversión exterior } \\
\text { Cuenta de pérdidas y ganancias } \\
\text { Empleo de calidad }\end{array}$ & $\begin{array}{l}\text { ¿Cómo lo medimos? } \\
\text { ¿Están definidos los indicadores de resultado por instrumento de } \\
\text { apoyo? } \\
\text { ¿Cómo se vinculan estos indicadores con los indicadores de objetivos } \\
\text { reflejados en el plan estratégico de la Secretaría de Estado de } \\
\text { Comercio? }\end{array}$ \\
\hline Impacto & Retorno de inversión vía impuestos & ¿Cuánta riqueza apalancan los recursos que dedicamos? \\
\hline
\end{tabular}

\section{Beneficiarios}

Para los beneficiarios de los apoyos, los clientes de nuestros servicios, la pregunta básica es saber qué perfiles de empresa recurren a cada apoyo. Utilizando tanto las respuestas recibidas vía encuesta en un bloque que llamamos características de empresa, como los valores de ciertas magnitudes del registro mercantil, realizamos una segmentación de clientes mediante técnicas de clusterización, normalmente jerárquica, a partir de variables categóricas $u$ ordinales. Todas estas segmentaciones han resultado ser robustas y han mostrado tipologías de empresas que funcionan bien como diferentes niveles de una variable sintética de estrato que logra separar de manera coherente los diferentes logros mostrados.

Con posterioridad, hemos utilizado estos clústeres robustos obtenidos como variable objetivo para construir clasificadores que predigan el clúster de pertenencia de una empresa cualquiera. Para este fin, utilizamos todos los tipos de clasificador que permite el espacio topológico definido por las variables explicativas, y seleccionamos el mejor según el doble criterio de minimizar la tasa de error con la mayor limpieza medida mediante el área bajo la curva ROC (receiver operating characteristic). Hasta ahora, todos nuestros modelos de clasificación tienen una precisión de entre el $79 \%$ y el $99,5 \%$, con un área bajo curva ROC superior a 0,96 (lo que implica una gran limpieza en la clasificación, en términos de que no puede mejorarse la clasificación con la información disponible), y debemos destacar que las variables más relevantes son las capturadas mediante nuestras encuestas de evaluación, pues, al prescindir de dichas variables, la precisión desciende hasta el $54 \%$ en el mejor de los casos, o, lo que es lo mismo, la tasa de error sube como mínimo al $46 \%$.

\section{Tratamiento}

El servicio que ofrece ICEX es, de forma genérica, apoyo público a la internacionalización de la empresa. Las preguntas que surgen son en qué consiste este apoyo y si puede considerarse un apoyo puntual o un acompañamiento continuado. En este último caso, la pregunta inmediata es si las diferentes tipologías de empresa que acuden a nosotros siguen alguna $D$ 
TABLA 8

ALGUNOS EJEMPLOS DE CLASIFICADORES

\begin{tabular}{|c|c|c|c|c|}
\hline & Clasificador 1 & Clasificador 2 & Clasificador 3 & Clasificador 4 \\
\hline Tipo & Necesidades & Necesidades & Efectividad & General \\
\hline $\begin{array}{l}\text { Modelo de } \\
\text { clasificación }\end{array}$ & Regresión logística & $\begin{array}{l}\text { Potenciación de } \\
\text { gradientes }\end{array}$ & Regresión logística & Regresión logística \\
\hline Objeto & Ferias de Pabellón Oficial & $\begin{array}{l}\text { Apoyo a acciones } \\
\text { comerciales }\end{array}$ & SSPP & Perfiles de empresa \\
\hline $\begin{array}{l}\text { Población } \\
\text { representada }\end{array}$ & $\begin{array}{l}\text { Empresas usuarias del apoyo a la } \\
\text { participación en Ferias de Pabellón Oficial }\end{array}$ & $\begin{array}{l}\text { Empresas usuarias de } \\
\text { servicios de apoyo }\end{array}$ & $\begin{array}{l}\text { Empresas usuarias } \\
\text { de SSPP }\end{array}$ & $\begin{array}{l}\text { Empresas usuarias } \\
\text { de servicios de ICEX }\end{array}$ \\
\hline Variables & 7 & 11 & 7 & 65 \\
\hline Dimensiones & 3 & 4 & 3 & 22 \\
\hline Precisión & $99 \%$ & $79 \%$ & $93 \%$ & $79 \%$ \\
\hline Limpieza & 1,000 & 0,973 & 0,991 & 0,995 \\
\hline
\end{tabular}

estrategia a la hora de solicitar apoyos que maximice su beneficio.

Para contestar estas preguntas aplicamos técnicas de análisis secuencial, tanto para todas las empresas como por cada una de las tipologías encontradas en cada programa de ICEX evaluado. El Gráfico 1 muestra los patrones genéricos del uso de categorías de instrumento entre 2003 y 2019. Por su parte, el Gráfico 2 muestra los patrones genéricos del $D$

GRÁFICO 1

PATRONES GENÉRICOS DE USO DE CATEGORÍAS DE INSTRUMENTO DE ICEX ENTRE 2003 Y 2019 PARA TODOS LOS CLIENTES

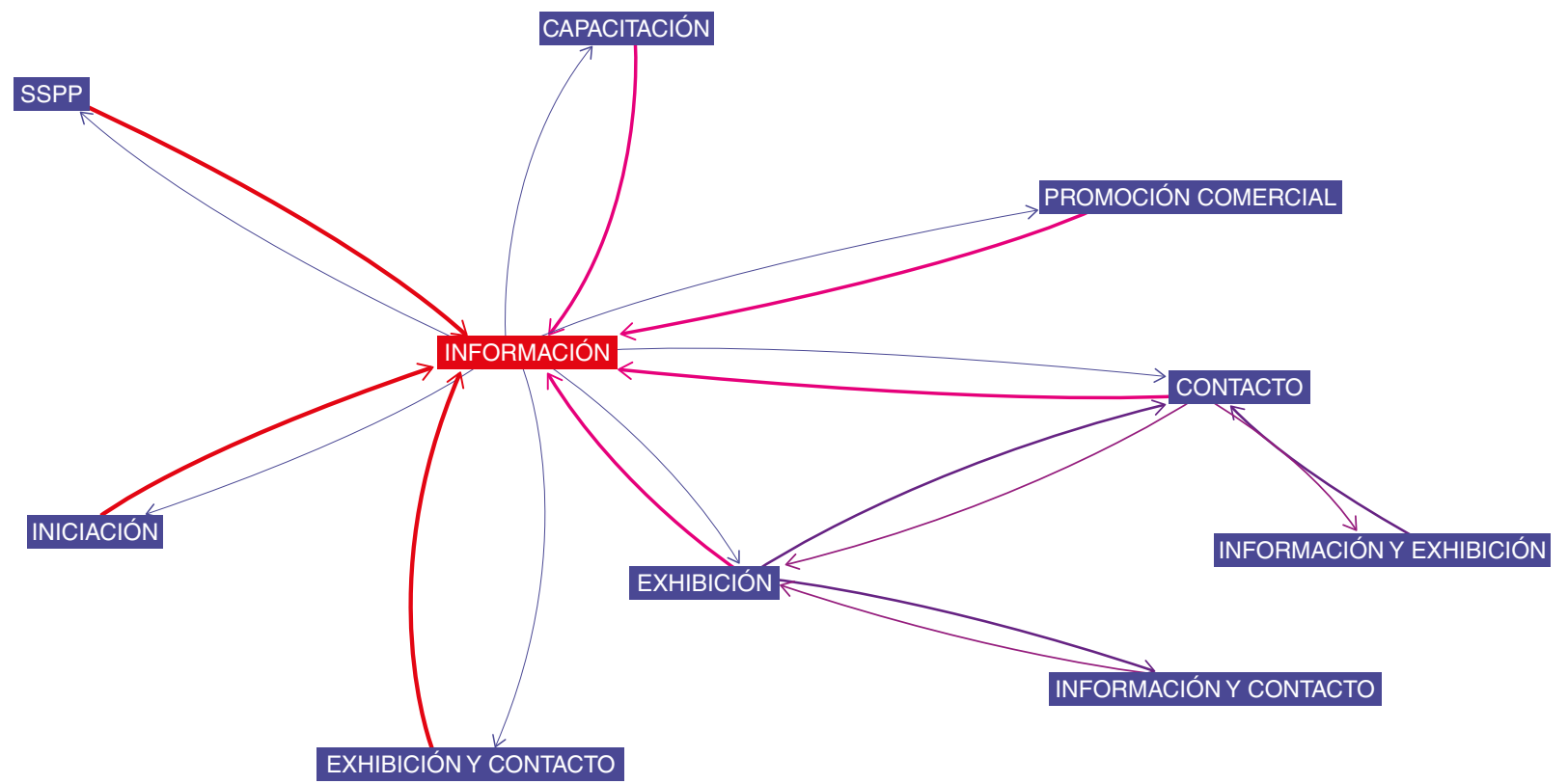

Datos: participación en categorías de instrumento de ICEX entre 2003 y 2019 por NIF. Tamaño del fichero: 465.566 registros (correspondientes al cruce NIF-Año-Categoría de instrumento).

Total de secuencias: 133.359.

Técnica: análisis secuencial.

Fuente: Departamento de Evaluación de ICEX. 


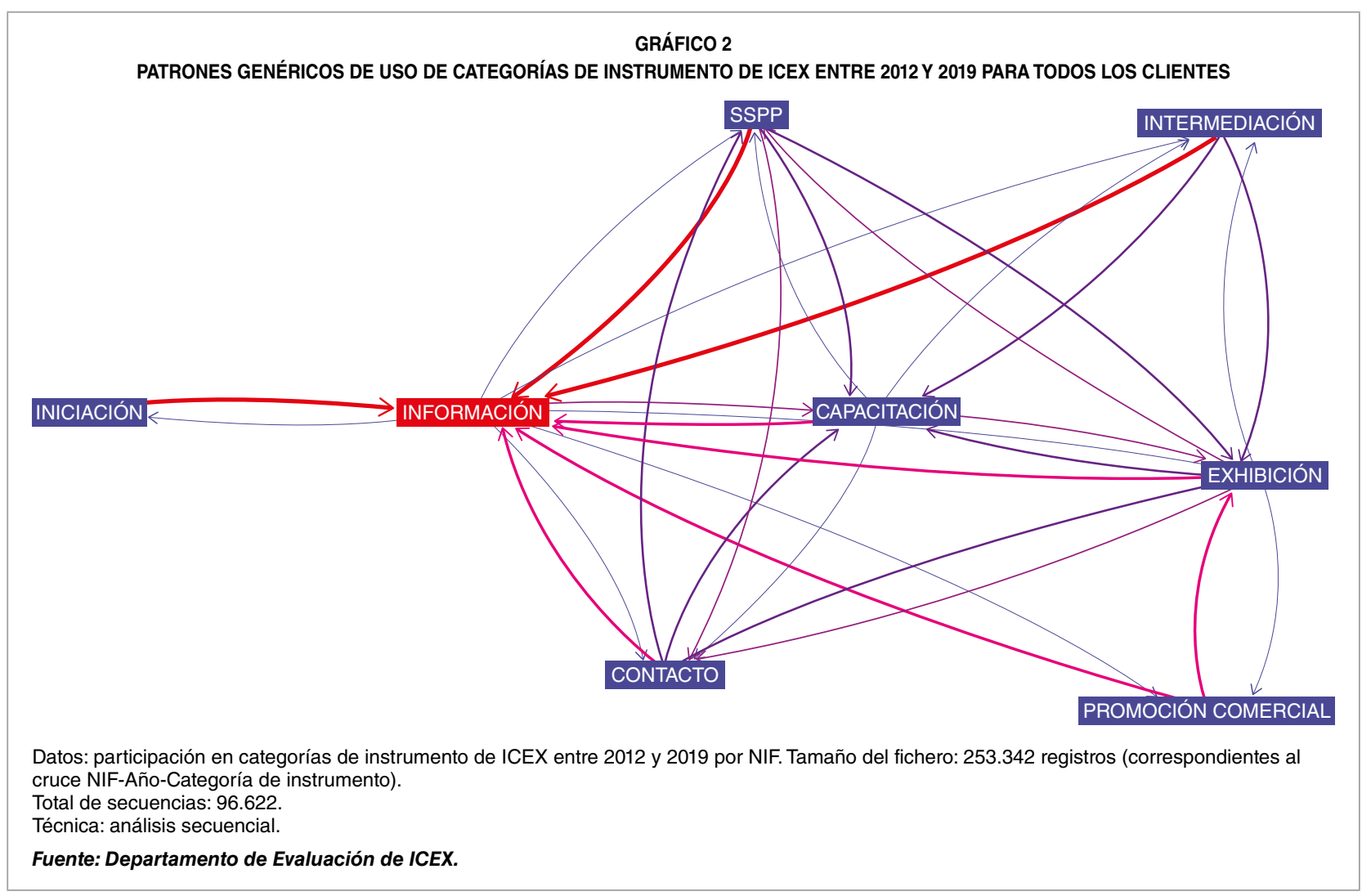

uso de categorías de instrumento entre 2012, año en que ICEX se convirtió en Ente Público Empresarial, y 2019. Por último, el Gráfico 3 muestra un diagrama de Sunkey de los patrones de uso de categorías tomando en cuenta solo el primer año de uso de cada categoría por la empresa, lo que permite ver de forma muy concisa las combinaciones más usuales de categorías de instrumento.

Los resultados de estos análisis han mostrado que tipologías de empresa distintas nos utilizan de forma diferente y nos permiten predecir con gran precisión cuál es el apoyo concreto más probable que una empresa nos va a solicitar en los siguientes dos años, conociendo la sucesión de apoyos que nos ha pedido en el pasado. Los modelos construidos permiten incluso predecir, para empresas que nunca nos han solicitado ningún apoyo, cuál es la puerta de entrada más probable a nuestra amplia cartera de servicios, en función de sus características.

\section{Producto}

Cuando llegamos al producto, la pregunta fundamental es qué nos piden las empresas. El bloque del cuestionario denominado preferencias de apoyo para superar barreras de mercado incluye unas preferencias fijas, que se muestran en la Tabla 2, si bien en algún caso se ha incluido alguna adicional de relevancia para el programa a evaluar.

En este bloque pedimos que las empresas listen por orden de preferencia los apoyos que, en función de sus necesidades, requieren de la Administración pública, lo que nos permite conocer las carencias de las empresas. El análisis de este bloque no está exento de caer en $\triangleright$ 


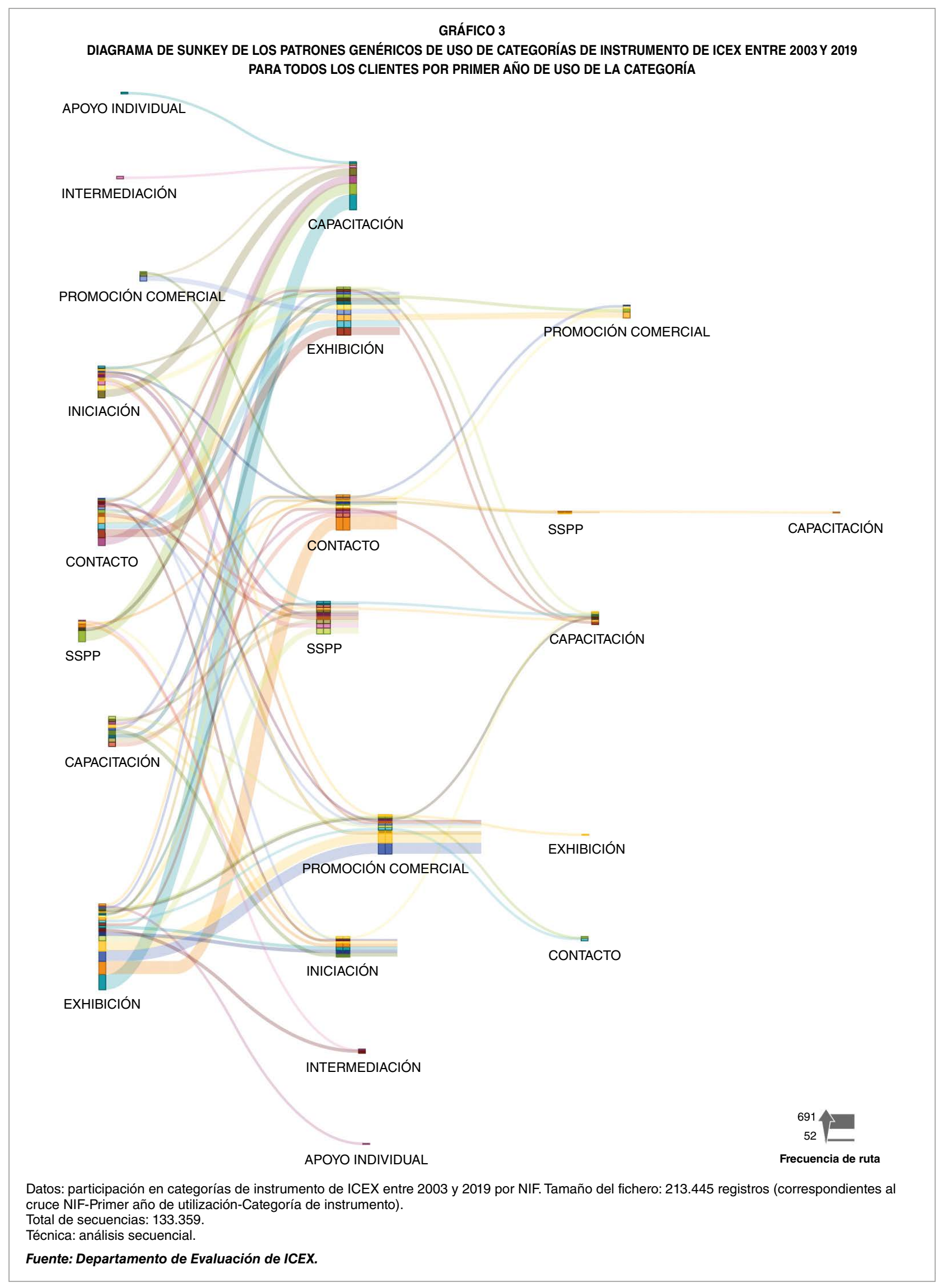




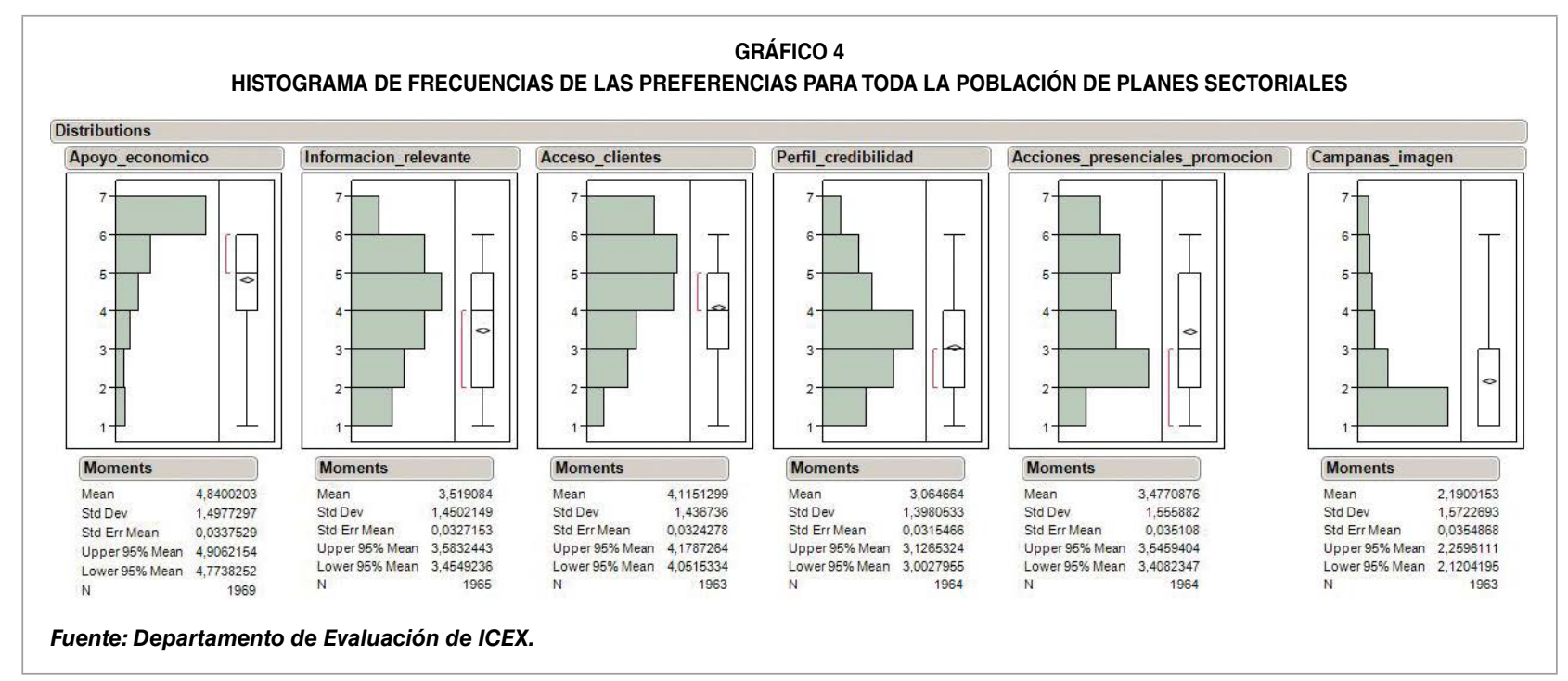

la paradoja de Condorcet, subyacente a toda función de preferencias generalizadas. Esta paradoja establece que, a veces, lo más votado es lo menos preferido. La pregunta que surge entonces es si las empresas realmente piden lo que necesitan, y nuestra preocupación como institución pública que debe promover la internacionalización de la empresa española es saber realmente qué necesitan para tener éxito en su singladura internacional.

Para ello, no nos contentamos con hallar la distribución de las posiciones otorgadas a cada preferencia por las empresas, pues esto puede llevar a conclusiones engañosas, sino que realizamos un análisis de preferencias mediante técnicas de escalamiento multidimensional, que nos muestra cómo dichas preferencias se asocian entre sí.

Como ejemplo, en el Gráfico 5 vemos que, para los clientes de los Planes Sectoriales, el apoyo económico y el perfil de credibilidad son excluyentes, igual que lo son el acceso a clientes y las acciones presenciales de promoción. Por otro lado, la información relevante es casi excluyente con las acciones presenciales de promoción y se opone en gran medida a las campañas de imagen. Atendiendo a los resultados del escalamiento y a la información de los histogramas, estas empresas, sobre todo, requieren el tándem de información relevante de difícil obtención y el acceso efectivo a clientes.

\section{Resultado}

Llegamos ahora a la parte del resultado de nuestros apoyos, definidos como los efectos inmediatos de los productos y servicios que ofrecemos a las empresas cliente. La pregunta que surge a este respecto es, en primer lugar, en qué incidimos y, en segundo lugar, comprobar la atribución de nuestro apoyo, es decir, el vínculo causal entre la intervención y los efectos observados.

Tras reconstruir la lógica de la intervención de cada apoyo, plasmamos las relaciones en un esquema que hace posible visualizar las posibles relaciones teóricas de causa y efecto entre las variables identificadas. Todas estas relaciones constituyen hipótesis que comprobamos mediante la ejecución del test de hipótesis apropiado.

Tal y como hemos dicho anteriormente, en esta primera fase hemos utilizado falsos $\triangleright$ 


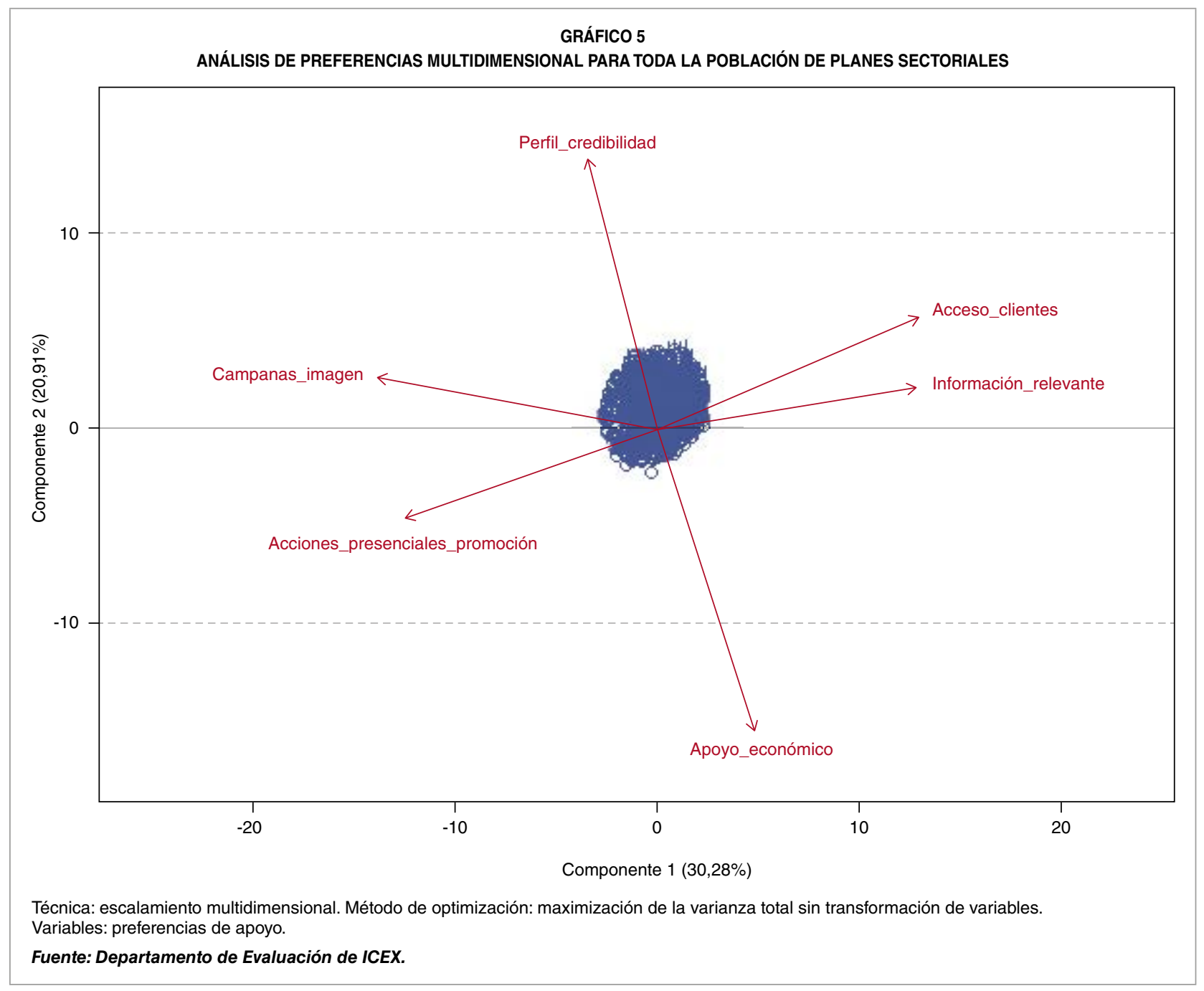

contrafactuales, es decir, hemos utilizado el propio grupo de tratamiento como grupo de control. En particular, hemos recurrido a la técnica de la pregunta implícita, que consiste en que la propia pregunta de la encuesta hace referencia al grado en que la empresa ha percibido que el apoyo ha incidido en diferentes aspectos de mejora de competitividad. Además, incluimos siempre un bloque que llamamos de valor añadido que consta de las dos preguntas implícitas siguientes.

Beneficios. Valore si los beneficios que le ha supuesto el uso del servicio o la participación en el programa son mayores al coste de su participación.
Adicionalidad. Valore su grado de acuerdo con la siguiente afirmación: «El apoyo prestado por ICEX le ha permitido lograr resultados que por sí solo no le hubiera sido posible conseguir».

La primera tiene que ver con la relación calidad-precio del apoyo público y la segunda, con el grado de adicionalidad que ha supuesto el apoyo público. Como estamos además interesados en conocer cuándo tenían lugar los efectos en nuestros clientes tras el apoyo prestado por ICEX, en nuestras evaluaciones realizamos un diseño muestral robusto y equilibrado que permite preguntar a diferentes cohortes de empresas beneficiarias del programa de $\triangleright$ 


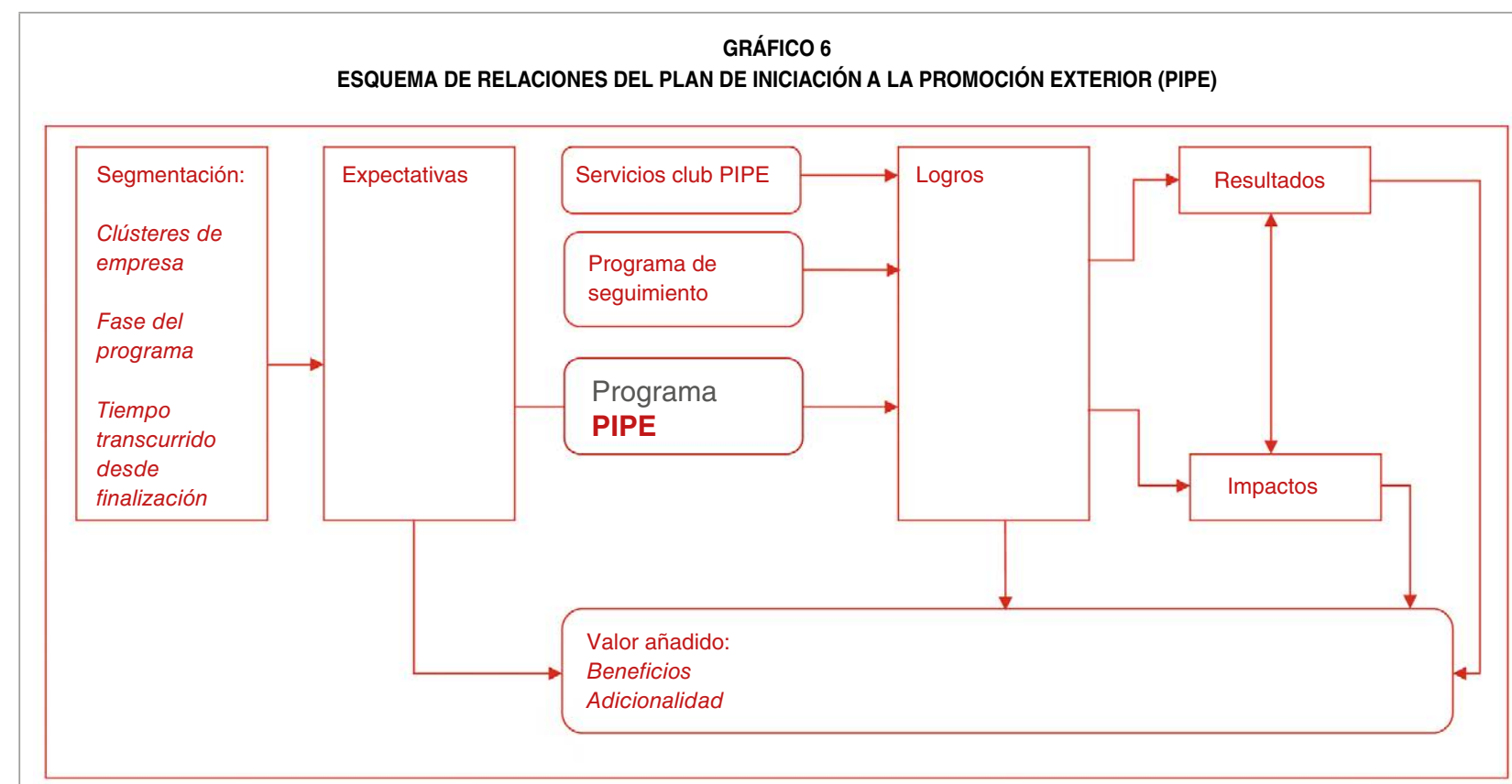

Fuente: Departamento de Evaluación de ICEX.

apoyo, como mínimo entre un año y tres años anteriores al ejercicio en que realizamos la evaluación, aunque en ocasiones hemos llegado a preguntar a empresas que recibieron el apoyo cinco años antes. Esto nos permite identificar efectos a lo largo del tiempo, como mínimo, entre uno y tres años después de la intervención. Como ejemplo, el Gráfico 9 muestra el diseño cuasiexperimental que subyacía a la evaluación del programa PIPE.

En primer lugar, contestamos la pregunta de qué efectos ha habido en la población tras la intervención pública mediante un análisis de la varianza multivariante junto con tests de $D$

\section{GRÁFICO 7}

DISEÑO CUASIEXPERIMENTAL SUBYACENTE EN LA EVALUACIÓN DEL PIPE

\begin{tabular}{|l|}
\hline EMPRESA ANTES \\
Expectativas \\
\hline PROGRAMA PIPE \\
Valor añadido: \\
Beneficios \\
Adicionalidad \\
\hline EMPRESA DESPUÉS \\
\hline Logros \\
Resultados \\
Impactos \\
\hline
\end{tabular}

\begin{tabular}{|c|c|c|c|c|c|c|c|}
\hline & 2006 & 2007 & 2008 & 2009 & 2010 & 2011 & 2012 \\
\hline NRAND & 01 & $X$ & $X$ & 02 & & & 03 \\
\hline NRAND & & 01 & $X$ & $X$ & 02 & & 03 \\
\hline NRAND & & & 01 & $X$ & $X$ & 02 & 03 \\
\hline
\end{tabular}

NRAND (NON-RANDOM): autoselección, asignación no aleatoria al grupo de estudio

X: aplicación de tratamiento: programa PIPE

O: observación de variables

01: expectativas antes de entrar en el programa

02: logros tras salir del programa

O3: impactos, resultados y valor añadido del programa

Fuente: Departamento de Evaluación de ICEX. 
Joaquín María Núñez Varo y María del Mar Gómez González

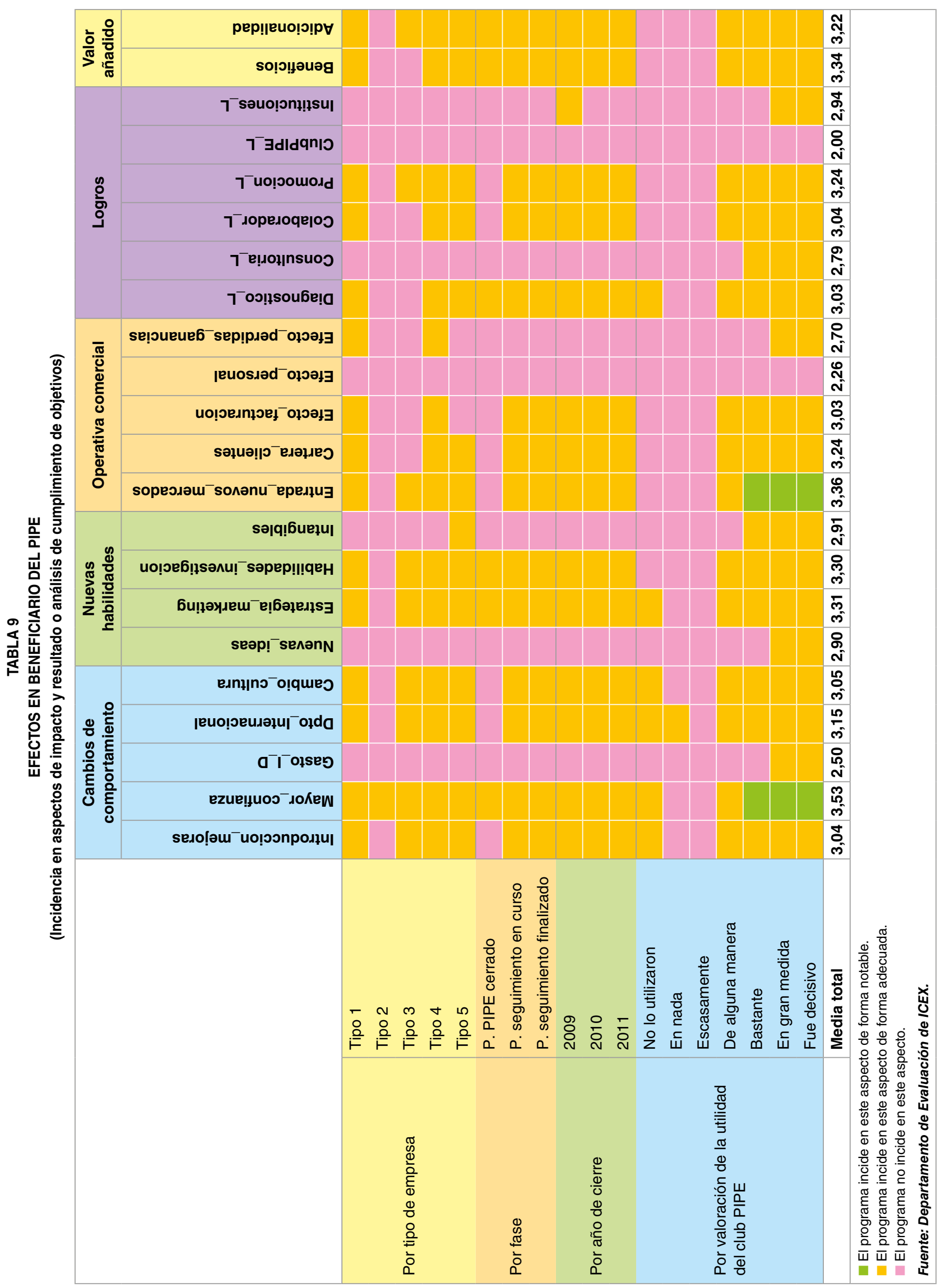


comparaciones múltiples de medias. Solemos utilizar principalmente los tests de Duncan y de Dunnett, en función de si un test previo de homogeneidad de la varianza nos permite suponer que las varianzas dentro de cada nivel en las variables de clasificación son homogéneas o no. Estos tests de comparaciones múltiples de medias nos indican si se puede dividir la población en tres grupos: uno cuya media esté por encima de la media poblacional; otro cuya media sea equivalente a la media poblacional; y un tercero cuya media sea inferior a la media poblacional. Tras tener la población dividida en estos grupos, los coloreamos en función de su media según las escalas de Likert y mostramos gráficamente, como recoge la Tabla 9, en qué variables ha habido efectos en las empresas cliente tras haber recibido el apoyo.

En estos gráficos buscamos, sobre todo, patrones verticales $u$ horizontales, o rupturas de patrones verticales u horizontales. Un patrón vertical implica que el efecto en un determinado aspecto de resultado es independiente de los diferentes niveles de la variable de clasificación, mientras que un patrón horizontal muestra que un determinado nivel de clasificación presenta un comportamiento diferenciado $y$, por tanto, propio de dicho nivel de clasificación. Las rupturas de patrones tienen una lectura similar: una ruptura de patrón vertical muestra que para un nivel determinado el comportamiento es distinto del resto de la población en un determinado aspecto, y una ruptura de patrón horizontal muestra que para un determinado nivel el comportamiento en algún aspecto es distinto de su comportamiento general.

Ahora bien, este análisis de incidencia en variables de impacto y resultado no implica que el efecto observado sea atribuible a nuestra intervención. Para ello, necesitamos realizar la técnica de estimación de impacto contemplada, que es una regresión logística que modelice la probabilidad de puntuar 4 o 5 (rango alto de una escala de Likert) en las variables de valor añadido en función de todas las variables de logros y efectos en aspectos de mejora de competitividad (nuestras variables de impactos y resultados) del cuestionario.

\section{Atribución de efectos al apoyo público: cadena de valor público}

Tras realizar esta regresión logística, construimos una tabla donde mostramos para qué variables un aumento o un descenso implica necesariamente una mayor probabilidad de puntuar 4 o 5 en las variables de valor añadido. La Tabla 10 muestra los resultados más interesantes para el programa PIPE, en particular la evolución del reconocimiento del valor del apoyo prestado según el paso del tiempo. En términos de adicionalidad, las empresas reconocen un año después de la terminación del programa dicho valor en la mejora en la estrategia de marketing. Dos años después, lo reconocen en el cambio de cultura integral en la empresa. Tres años después, el reconocimiento se plasma en la decisión de incrementar el gasto en I+D. Por su parte, en términos de beneficios (relación calidad-precio), las empresas reconocen los beneficios de haber participado en el programa un año después de la terminación del PIPE en una mayor confianza a costa de un empeoramiento de la cuenta de pérdidas y ganancias (lo que implica inversión). Dos años después, en la introducción de mejoras. Tres años después, el reconocimiento se plasma en el desarrollo de un Departamento Internacional y en un efecto positivo en la cuenta de pérdidas y ganancias. 
TABLA 10

ATRIBUCIÓN DE EFECTOS EN BENEFICIARIO AL PROGRAMA PIPE

(variables de adicionalidad y de beneficios)

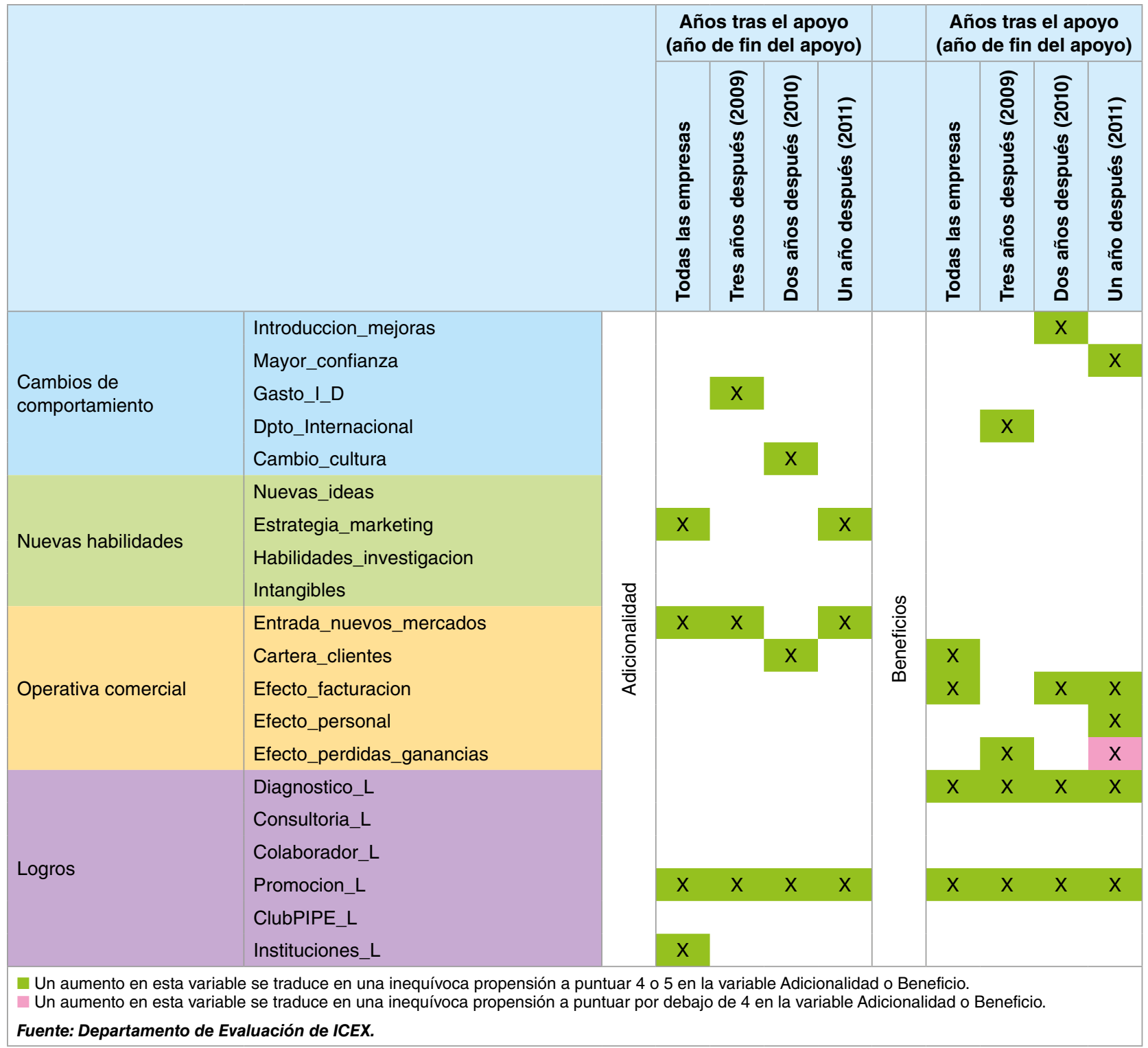

Aunque todas nuestras evaluaciones tienen un diseño elaborado que permite obtener resultados muy detallados, mostramos en la Tabla 11, a modo de resumen, el porcentaje de empresas que reconoce un grado adecuado, como mínimo, en el valor añadido del servicio recibido (sea en relación calidad-precio o en adicionalidad), estructurado por su tamaño, según los criterios de la UE. Disponer de esta información resulta clave a la hora de tomar decisiones relativas a los apoyos orientados a distintos segmentos de empresas, según su tamaño.

\section{Evaluaciones de necesidades}

Vamos a comentar brevemente cómo acometemos las evaluaciones de necesidades, que realizamos cuando queremos indagar $D$ 
TABLA 11

PORCENTAJE DE EMPRESAS QUE RECONOCE COMO MÍNIMO UN GRADO ADECUADO EN EL VALOR AÑADIDO DEL SERVICIO (SEA EN RELACIÓN CALIDAD-PRECIO O EN ADICIONALIDAD) POR SU TAMAÑO SEGÚN LOS CRITERIOS DE LA UE

\begin{tabular}{|l|c|c|c|c|}
\hline \multirow{2}{*}{\multicolumn{1}{|c|}{ Grupo actividad }} & \multicolumn{4}{c|}{ Tamaño de empresa } \\
\cline { 2 - 5 } & Microempresa & Pequeña empresa & Mediana empresa & No pyme \\
\hline Apoyo a inversión & $55,56 \%$ & $88,89 \%$ & $74,19 \%$ & $76,47 \%$ \\
Apoyo individual & $88,00 \%$ & $82,27 \%$ & $80,00 \%$ & $85,53 \%$ \\
Contacto & $81,97 \%$ & $79,08 \%$ & $83,96 \%$ & $88,57 \%$ \\
Exhibición & $77,24 \%$ & $80,92 \%$ & $83,76 \%$ & $-100,00 \%$ \\
Información & $50,00 \%$ & $100,00 \%$ & $96,08 \%$ & $100,00 \%$ \\
Iniciación & $84,00 \%$ & $85,48 \%$ & $82,35 \%$ & $76,92 \%$ \\
Relaciones públicas & $65,67 \%$ & $73,64 \%$ & $76,15 \%$ & $82,39 \%$ \\
Servicio personalizado & $70,76 \%$ & $75,32 \%$ & & - \\
\hline Nota: el análisis realizado en cada evaluación por segmentos de empresa y por tiempo en que se produce el efecto ofrece resultados más detallados. \\
\multicolumn{4}{|l}{ Fuente: Departamento de Evaluación de ICEX. }
\end{tabular}

qué tipo de apoyo tendríamos que dar a cada tipología de empresa, en función de sus necesidades y sus capacidades.

Estas evaluaciones constan de al menos una primera fase de la cual, mediante técnicas cualitativas (principalmente grupos de discusión y entrevistas en profundidad), extraemos ideas bien formuladas de apoyos que ICEX podría prestar y que son demandados con claridad por alguna empresa participante en el trabajo de campo, que es representativa, de acuerdo con el diseño de muestra calculado previamente, de un tipo de empresa definido. Diseñamos toda la evaluación y dirigimos el proceso, aunque subcontratamos la ejecución de las técnicas. Un ejemplo de la forma de proceder y los resultados que obtenemos en esas fases cualitativas puede verse en De Lucio (2018).

En una fase posterior, sometemos todas las propuestas identificadas al grueso de empresas que podrían estar interesadas en dichos servicios bajo una metodología clásica de test de producto, donde preguntamos por el interés en la propuesta de servicio y por su intención de precio, y analizamos luego las respuestas mediante una metodología cuantitativa propia.

\section{Autodiagnóstico de las capacidades de la empresa}

En todas las evaluaciones de necesidades incluimos un bloque de diagnóstico de la empresa, donde preguntamos acerca de sus capacidades generales de producción, de sus recursos humanos y de su departamento comercial. Para analizar este bloque, usamos una técnica para extraer la preferencia en diferentes ítems probada en sistemas de recomendación, que evitan el problema de los diversos umbrales de percepción propios para cada empresa, es decir, la divergencia en los grados de exigencia y de referencia de empresas que se encuentren en diferente estado de madurez y desarrollo. En concreto, tipificamos las puntuaciones de cada empresa de acuerdo con la distribución de sus calificaciones en todas las variables de diagnóstico y sometemos estas nuevas variables tipificadas a un análisis de escalamiento dimensional. Como resultado, hemos identificado tres ejes, que llamamos factores de oposición, y la posición a lo largo de estos ejes clasifica cada empresa en términos de sus capacidades reales. Cada uno de estos ejes, que detallamos a continuación, $D$ 
está constituido a su vez por dos dimensiones distintas en pugna.

- El eje 1 muestra el equilibrio entre las siguientes dimensiones:

- Cultura internacional y red de contactos.

- Recursos humanos y financieros.

- El eje 2 muestra el equilibrio entre las siguientes dimensiones:

- Formación continua y capacidad analítica.

- Labores tradicionales de un departamento comercial.

- El eje 3 muestra el equilibrio entre las siguientes dimensiones:

- Recursos financieros y capacidad productiva.

- Talento de los recursos humanos comerciales.
Llamamos palanca de competitividad a aquella dimensión dentro de cada eje que está reforzada en cada tipo de empresa. La Tabla 12 muestra las palancas de competitividad de los ocho tipos distintos de empresa surgidos de la evaluación de necesidades en apoyos a acciones de prospección comercial en el mercado internacional.

\section{Análisis del interés en las propuestas de servicio}

Las empresas, a continuación, pueden valorar su interés en cada propuesta de servicio identificada mediante una escala de Likert, e indicar una intención de precio en función de ciertas horquillas preestablecidas. Calculamos para las variables de interés la desviación tipificada con respecto al valor medio de todas las variables de interés en las mejoras de cada observación, e igualmente para las variables de intención de precio. $\triangleright$

TABLA 12

PALANCAS DE COMPETITIVIDAD PARA LOS DIFERENTES TIPOS DE EMPRESA QUE SOLICITAN APOYO A SUS ACCIONES INDIVIDUALES DE PROSPECCIÓN COMERCIAL

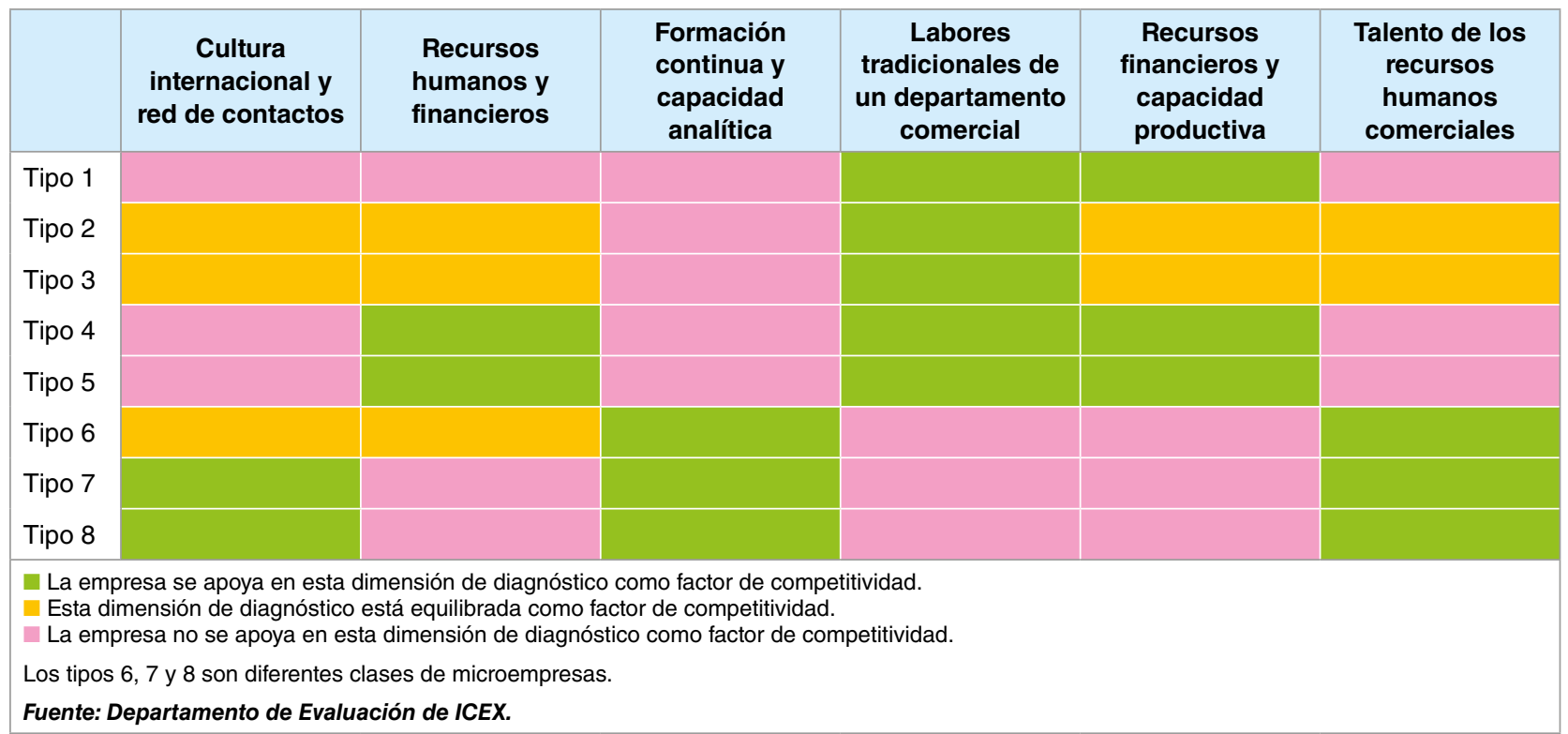


A continuación, sometemos tanto las variables originales como las tipificadas a un análisis de colas de distribución, tanto según la distribución dentro del propio tipo de empresa como entre tipos de empresa, y triangulamos estos resultados con los obtenidos en la fase cualitativa. La Tabla 13 muestra, a modo de ejemplo, los resultados solo del análisis cuantitativo para la propuesta «Información especializada por sector». A la hora de interpretar estos resultados, es conveniente tener en cuenta que, dada la naturaleza de los datos, para la población con escasa intención de precio, el valor reflejado en los cuadros sobreestima el valor real de la media de esta población, mientras que, para aquella con alta intención de precio, el valor mostrado lo estará subestimando.

A partir de la información recabada en la evaluación, incluyendo el diagnóstico, el interés en propuestas, y la sucesión de apoyos que ha solicitado la empresa de ICEX, podemos hacernos una idea bastante precisa de qué requiere cada tipo de empresa y en qué deberíamos ayudarla, pues además podemos conocer, a partir de los resultados de las evaluaciones de impacto, la efectividad del apoyo que podríamos ofrecerle, si la categoría de apoyo a la que pertenece ha sido previamente evaluada.

TABLA 13

INTERÉS E INTENCIÓN DE PRECIO EN LA PROPUESTA DE OFRECER SERVICIOS DE INFORMACIÓN ESPECIALIZADA

\begin{tabular}{|c|c|c|c|c|c|c|}
\hline & Interés (1) & $\begin{array}{l}\text { Interés. } \\
\text { Desviación } \\
\text { respecto a } \\
\text { media (2) }\end{array}$ & $\begin{array}{l}\text { Interés. } \\
\text { Desviación } \\
\text { respecto a } \\
\text { clúster (3) }\end{array}$ & Precio (4) & $\begin{array}{l}\text { Precio. } \\
\text { Desviación } \\
\text { respecto a } \\
\text { media (5) }\end{array}$ & $\begin{array}{l}\text { Precio. } \\
\text { Desviación } \\
\text { respecto a } \\
\text { clúster (6) }\end{array}$ \\
\hline Tipo 1 & 4,12 & $93 \%$ & $93 \%$ & 271 & $79 \%$ & $79 \%$ \\
\hline Tipo 2 & 3,97 & $74 \%$ & $74 \%$ & 177 & $59 \%$ & $59 \%$ \\
\hline Tipo 3 & 3,88 & $74 \%$ & $74 \%$ & 180 & $62 \%$ & $62 \%$ \\
\hline Tipo 4 & 3,98 & $85 \%$ & $85 \%$ & 187 & $73 \%$ & $73 \%$ \\
\hline Tipo 5 & 3,91 & $77 \%$ & $77 \%$ & 198 & $66 \%$ & $66 \%$ \\
\hline Tipo 6 & 3,85 & $60 \%$ & $60 \%$ & 172 & $53 \%$ & $53 \%$ \\
\hline Tipo 7 & 3,93 & $73 \%$ & $73 \%$ & 157 & $42 \%$ & $42 \%$ \\
\hline Tipo 8 & 3,78 & $70 \%$ & $70 \%$ & 135 & $38 \%$ & $38 \%$ \\
\hline p-valor (ANOVA multiv.) & 0,09 & 0,00 & 0,00 & 0,00 & 0,00 & 0,00 \\
\hline Media total & 3,93 & & $76 \%$ & 189 & & $60 \%$ \\
\hline \multicolumn{7}{|l|}{ Código de lectura. } \\
\hline \multicolumn{7}{|c|}{$\begin{array}{l}\text { Columna 1: Interés en la propuesta. } \\
\text { Esta mejora despierta un interés notable. } \\
\text { Esta mejora despierta interés. } \\
\text { Esta mejora despierta poco interés. }\end{array}$} \\
\hline \multicolumn{7}{|c|}{$\begin{array}{l}\text { Columna 4: intención de precio. } \\
\text { La intención de precio para esta mejora es superior a la media. } \\
\text { La intención de precio para esta mejora es indistinguible de la media. } \\
\text { La intención de precio para esta mejora es inferior a la media. }\end{array}$} \\
\hline \multicolumn{7}{|c|}{$\begin{array}{l}\text { Columna 2: Interés en la propuesta y } 5 \text { : intención de precio. } \\
\text { El valor se encuentra en la cola superior de la distribución para este tipo de empresa. } \\
\text { El valor se encuentra dentro del grueso de la distribución para este tipo de empresa. } \\
\text { El valor se encuentra en la cola inferior de la distribución para este tipo de empresa. }\end{array}$} \\
\hline \multicolumn{7}{|c|}{$\begin{array}{l}\text { Columna 3: Interés en la propuesta y 6: intención de precio. } \\
\text { El valor se encuentra en la cola superior de la distribución entre tipos de empresa. } \\
\text { El valor se encuentra dentro del grueso de la distribución entre tipos de empresa. } \\
\text { El valor se encuentra en la cola inferior de la distribución entre tipos de empresa. }\end{array}$} \\
\hline \multicolumn{7}{|c|}{ Fuente: Departamento de Evaluación de ICEX. } \\
\hline
\end{tabular}




\section{Perfilado de empresas}

Hace algunos años empezamos a trabajar en la creación de un índice sintético del potencial de internacionalización de una empresa. Como primer paso, en 2017 se realizó un primer estudio para identificar perfiles de las empresas que habían acudido a ICEX entre 2009 y 2016, teniendo en cuenta 65 variables de caracterización y competitividad que un análisis factorial mostró que representaban diversas facetas de 22 factores latentes. Este estudio reveló diez grupos de empresas muy distintos, para los que es posible inferir necesidades diferenciadas en materia de internacionalización. En la actualidad, el ejercicio se está ampliando a empresas usuarias de servicios de ICEX entre 2003 y 2019 (más de 180.000 entidades entre diferentes formas societarias de empresas y autónomos registrados). Este estudio nos va a permitir determinar una segmentación de empresas en función de sus necesidades y potencial de internacionalización y refinar los clasificadores para identificar empresas a las que ofrecerles aquellos de nuestros servicios que solventen sus necesidades actuales o futuras. Dentro de este trabajo, en primer lugar, tuvimos que completar mediante imputación los datos recabados de Informa para 27 variables objetivas longitudinales a partir de 2008. En particular, se aplicaron el método de la maximización de la esperanza y el método de imputación por especificación completamente condicional (método de Monte Carlo basado en cadenas de Markov, MCMC), que daban resultados muy similares, y a continuación, como regla de decisión final para la imputación efectiva de cada valor faltante concreto, calculamos la combinación lineal aleatorizada entre ambos métodos mediante parámetro de distribución uniforme. La calidad de esta imputación se muestra en la Tabla 14.

\section{Sistema de recomendación basado en inteligencia artificial}

Todo el conocimiento extraído, que hemos intentado difundir de forma breve en este artículo, nos ha permitido plantear un sistema de recomendación que puede proponer, de manera anticipada, las actividades e instrumentos de apoyo de interés para el cliente en términos de efectividad del mismo y del valor de la acción pública. La retroalimentación de este sistema con los datos de efectividad real le hará ir ajustándose solo, por lo que entrará en el paradigma denominado inteligencia artificial. El Gráfico 8 muestra los bloques y flujos del sistema de recomendación diseñado, en cuya prueba estamos ahora mismo inmersos y plenamente ilusionados, pues supone un paso clave para conseguir que las evaluaciones que hemos acometido no supongan solo ejercicios teóricos, sino que permitan, en la práctica, mejorar la precisión y calidad del servicio que ofrecemos a la empresa.

TABLA 14

CALIDAD DE LA IMPUTACIÓN DEL CONJUNTO DE VARIABLES SELECCIONADAS DEL REGISTRO MERCANTIL PARA LAS EMPRESAS CLIENTE DE ICEX DESDE 2003

\begin{tabular}{|l|c|}
\hline \multicolumn{1}{|c|}{ Criterio de validez } & Valor \\
\hline Máximo error de imputación & $3 \%$ \\
Máxima variación en entropía individual y condicional & $1 \%$ \\
Máxima variación en información mutua entre componentes principales no redundantes & $1 \%$ \\
\hline Fuente: Departamento de Evaluación de ICEX. & \\
\hline
\end{tabular}


La implementación de este sistema de recomendación constituye uno de los proyectos prioritarios, que irá concretándose en los próximos meses mediante una mayor integración de los sistemas de información para facilitar y extender su uso y para iterar mediante la retroalimentación que permita ir mejorando progresivamente su utilidad y fiabilidad a través de la experiencia real.

\section{Conclusiones}

En un entorno institucional y social de creciente sensibilidad y orientación hacia la mejora de las políticas públicas, los procesos de evaluación han pasado en muchos casos de ser una mera obligación a convertirse en un eje estratégico. En ICEX, el extenso y exhaustivo trabajo desarrollado durante la última década ha sentado las bases para configurar a la evaluación como un potente vector de mejora interna que permita optimizar la gestión, potenciar el enfoque al cliente y promover la toma de decisiones encaminada a la consecución de los objetivos estratégicos marcados.

Los retos estructurales relacionados con las características del sector exterior español y la orientación estratégica, recogida tanto en el Plan de Acción para la Internacionalización de la Economía Española como en el Plan Estratégico de ICEX, definen claramente una hoja de ruta en un marco temporal concreto, si bien la coyuntura económica y los nuevos acontecimientos que se suceden nos abocan a escenarios de incertidumbre y a contextos presupuestarios dinámicos que plantean retos adicionales a los que es preciso ir dando respuesta de manera flexible y progresiva.

Para cumplir con nuestra misión de impulsar la internacionalización de la economía española, adquieren aún mayor relevancia tanto la cultura de orientación al cliente, poniendo un especial foco en las pymes, como la gestión $\square$

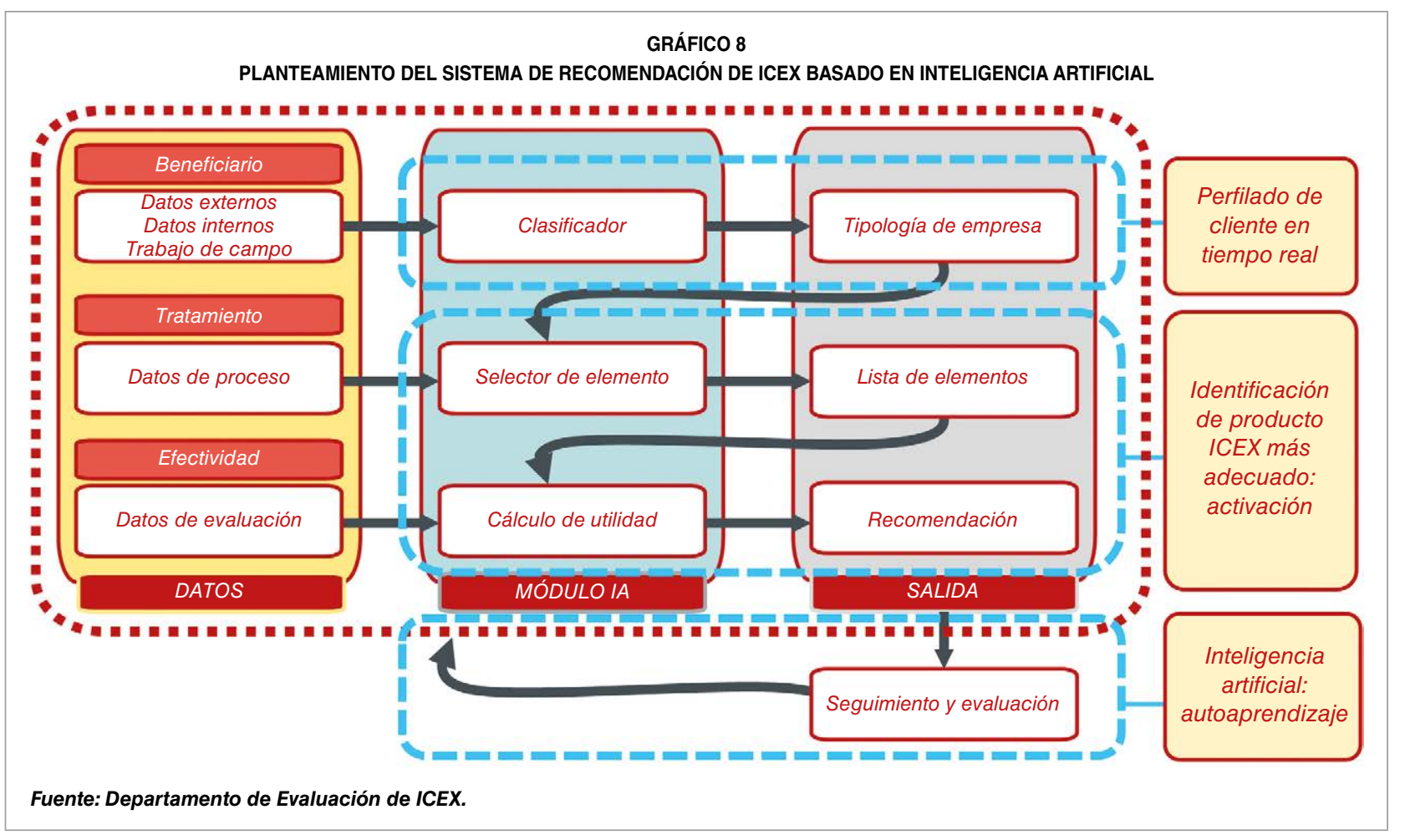


interna y optimización de los apoyos de ICEX. En este sentido, la aplicación de técnicas de ciencia de datos a los métodos tradicionales de evaluación, así como una visión analítica e integral de los apoyos que se prestan se configuran como palancas esenciales para impulsar las decisiones estratégicas en ICEX.

El desarrollo alcanzado por el equipo de evaluación interno, apoyado puntualmente con expertos y profesionales externos, ha conducido a un grado de conocimiento tanto del impacto de los programas y actividades como del perfilado de clientes y de las recomendaciones más adecuadas según sus necesidades, que coloca a ICEX en la mejor posición para proporcionar a la empresa un apoyo progresivamente más eficaz a lo largo de su proceso de internacionalización.

Todo ello no hubiera sido posible sin el impulso que ha supuesto el proyecto CRM Global ICEX durante la última etapa, como plataforma de información de los clientes y de su interacción con nosotros, junto con el intenso trabajo de Gobierno del Dato enfocado a mejorar paulatinamente la calidad de la información y los datos disponibles, así como el soporte técnico de alto nivel que proporciona Tecnologías de la Información. No en vano, disponer de información fiable y actualizada resulta esencial para poder realizar análisis precisos y extraer conclusiones que apoyen la toma de decisiones.

Nuevos proyectos y perspectivas se vislumbran en el horizonte cercano, ya que, junto con el sistema de recomendación enunciado, se abre igualmente la posibilidad de orientar la navegación en nuestro sistema de portales a través de la ludificación de la experiencia del usuario para poder conducirlo hacia el producto o servicio que mejor se adapte a sus necesidades e intentar promover que lo adquiera, mediante la contratación de un servicio, la descarga de información y contenidos relevantes o la inscripción en alguna actividad de su interés o adaptada a su perfil. En esencia, este proceso viene guiado por la siguiente relación:

Producto $=F$ (Necesidades, Efectividad, Disponibilidad)

Es decir, el producto que se debe ofrecer depende de la necesidad de la empresa, de la efectividad del producto en la propia empresa y de la disponibilidad del mismo dentro del catálogo y del plan de actividades de ICEX. Nuestra labor de evaluación ofrece evidencia robusta y relevante para poder contestar con fiabilidad las dos primeras cuestiones, y con unas pocas preguntas podríamos llevar a cualquier empresa que navegue por nuestros portales donde le resulte más oportuno. La Tabla 15 muestra el máximo número de preguntas necesarias para poder determinar el producto o servicio más apropiado para una empresa. Se trata de un número máximo de cuestiones, acotado y bastante asumible, pues no tiene sentido solicitar a una empresa información que ya conocemos. Este es el premio y la ventaja de aunar el conocimiento de la evaluación con la estrategia del dato.

Todos los grandes proyectos estratégicos precisan de la conjunción de elementos clave para su singladura y el caso de la evaluación en ICEX no ha sido una excepción. La combinación entre el impulso firme y decidido de todo el equipo, el compromiso con la mejora continua de la gestión interna que promueva la consecución de los objetivos definidos en el Plan Estratégico y un equipo altamente cualificado capaz de diseñar modelos de gran envergadura y extraer conclusiones rigurosas han resultado esenciales para consolidar progresivamente una ambiciosa estrategia en materia de evaluación.

Otro hito representa el Sistema de Seguimiento de Indicadores del Plan Estratégico $\triangleright$ 


\section{EL VALOR ESTRATÉGICO DE LA EVALUACIÓN EN ICEX}

TABLA 15 NÚMERO DE BITS (PREGUNTAS BINARIAS SÍ/NO) NECESARIAS PARA DETERMINAR EL PRODUCTO MÁS APROPIADO PARA UNA EMPRESA

\begin{tabular}{|c|c|c|c|c|}
\hline & Expresión & Necesidades (1) & Mercados (2) & Categorías de instrumentos (3) \\
\hline Número de posibles valores & $\mathrm{n}$ & 8 & 21 & 8 \\
\hline Bits & $\log _{2} n$ & 3 & 4,39 & 3 \\
\hline Preguntas necesarias & Techo( $\left(\log _{2} n\right)$ & 3 & 5 & 3 \\
\hline \multicolumn{5}{|c|}{$\begin{array}{l}\text { (1) Grupos de empresas según la evaluación de necesidades de apoyos a la prospección comercial. } \\
\text { (2) Zonas geográficas que definen mercados regionales. } \\
\text { (3) Número de categorías de instrumento de ICEX en función del producto o servicio ofrecido a la empresa. } \\
\text { Como las preguntas no son independientes, el número de preguntas en fases posteriores se reducen. Por ejemplo, concretar una necesidad implica } \\
\text { una reducción del conjunto de categorías que se puede ofrecer. } \\
\text { La efectividad se calcula en función de los parámetros anteriores y del tipo de la empresa. }\end{array}$} \\
\hline \multicolumn{5}{|c|}{ Fuente: Departamento de Evaluación de ICEX. } \\
\hline
\end{tabular}

de ICEX, una potente herramienta diseñada igualmente en el área de Evaluación que proporciona una visión integral del grado de cumplimiento de los objetivos de nuestra actividad medidos en diversos indicadores económicos clave, lo que se conoce en terminología de la Comisión Europea como el marco de rendimiento.

Finalmente, en el marco del firme compromiso de ICEX con el impulso a la internacionalización de las empresas y de la economía española, el refuerzo de la colaboración institucional en el seno de la Administración que facilite la puesta en común de datos e información clave con la finalidad de impulsar proyectos de evaluación, entendemos que abre las puertas a plantear posibles iniciativas conjuntas con objetivos aún más ambiciosos que promuevan análisis transversales, que aporten conclusiones de interés sobre la efectividad e impacto de los apoyos y que contribuyan a fomentar y optimizar la eficacia, el valor y la adicionalidad de las políticas públicas al servicio de la internacionalización en su conjunto.

\section{Bibliografía}

Aggarwal, C. C. (2016). Recommender Systems: The Textbook. Springer. https://link.springer.com/ content/pdf/10.1007\%2F978-3-319-29659-3.pdf
De Lucio (2018). Capítulo sobre necesidades de las empresas en su proceso de internacionalización. En P. de Diego, J. de Lucio, M. Martínez y J. Núñez, La internacionalización de la empresa española: Situación y propuestas. Civitas. https://www.thomsonreuters.es/es/tienda/duopapel-ebook/la-internacionalizacion-de-laempresa-espanola--situacion-y-propuestas-duo/ $\mathrm{p} / 10012891$

Gertler, P. J., Martínez, S., Premand, P., Rawlings, L. B., \& Vermeersch, C. M. J. (2016). Impact Evaluation in Practice. Word Bank (2nd. Edition). https://openknowledge.worldbank.org/ handle/10986/ 25030

HM Treasury (2011). The Magenta Book: Guidance for evaluation. https://www.gov.uk/government/ publications/the-magenta-book

Núñez, J. (2017). La evaluación del apoyo público a la internacionalización empresarial. (Disponible en la red Academia, mayo 2017). https://www. academia.edu/37555717/La_evaluaci\%C3\% B3n_del_apoyo_p\%C3\%BAblico_a_la_internacionalizaci\%C3\%B3n_empresarial

Peña, D. (2002). Análisis de datos multivariantes. McGraw-Hill. https://www.mheducation.es/analisis-multivariante-de-datos-9788448136109spain-group

Pérez Lopez, C., y Moral Arce, I. (2015). Técnicas de evaluación de impacto. Garceta.

Rincón-Aznar, A., Riley, R., \& Rosso, A. (2015). Evaluating the impact of UKTI trade services on the performance of supported firms. Report to the UKTI, the National Institute for Economic $\triangleright$ 
Joaquín María Núñez Varo y María del Mar Gómez González

and Social Research. https://www.gov.uk/government/publications/evaluating-the-impact-of-uktitrade-services-on-the-performance-of-supported-firms

Subdirección General de Estrategia de Internacionalización (2019). El Plan de Acción para la Internacionalización de la Economía Española 2019-2020. Boletín Económico de ICE (3111). https://doi.org/10.32796/bice.2019.3111.6827
Tan, P., Steinbach, M., Karpatne, A., \& Kumar, V. (2019). Introduction to Data Mining. Pearson (Second Edition). https://www-users.cs.umn. edu/ kumar001/dmbook/index.php

UKTI (2014). Technical Note: UKTI's monitoring and evaluation evidence. https://www.gov.uk/government/publications/technical-note-uktis-monitoring-and-evaluation-evidence/technical-noteuktis-monitoring-and-evaluation-evidence 